\title{
Detection of water condensation on exhaust gas sensors
}

Daniela Schönauer, Ralf Moos

Universität Bayreuth

Lehrstuhl für Funktionsmaterialien

95440 Bayreuth, Germany
Andreas Nauwerck, Till Gysin

Abgaszentrum der Automobilindustrie

c/o Dr. Ing. h. c. F. Porsche AG

Porschestraße

71287 Weissach, Germany

\section{Abstract}

An interdigital $\mathrm{H}_{2} \mathrm{O}$ sensing structure applied on a ceramic element provides the possibility to determine the water condensation process in the exhaust system of an internal combustion engine during cold-start. In lab tests, a clear correlation between capacity change and water droplet size was found. In engine cold start tests it was proven that the start and the end points of the water condensation processes can be detected.

\section{Introduction}

To minimize vehicle emissions, catalysts and exhaust gas sensors are integrated in the exhaust system. Both elements have to be heated quickly to working temperature. Although the ceramic sensor elements are protected by a metallic sensor housing consisting of protection tubes with bores for gas inflow [1], it is a serious issue of the elements that they are prone to be damaged by condensed water or water droplets (water splash) during engine cold start. Condensation occurs in the exhaust tube wall, in the sensor housing, and on the sensor element itself. If condensed water impinges on the heated ceramic sensor element, resulting thermal stress may lead to failures. Protective elements are discussed to minimize these effects [2].

This work focuses on the water condensation detection on a ceramic sensor element to minimize the failure rate of exhaust sensors and to provide fast sensor heating, already before reaching the end of the dew point.

\section{Experimental}

An interdigital electrode structure (IDE) was applied on an alumina substrate by screen-printing a platinum layer and subsequent laser patterning. Functionality tests are conducted by applying water droplets on the IDE, measuring the drop size and taking complex impedance data $I \underline{Z} I$ at $300 \mathrm{kHz}$. Engine cold start runs completed the test series. 


\section{Results and Discussion}

Initial impedance measurements with water droplets on top of an IDC structure show a typical semi-circle characteristic in the complex Nyquist Plot $(\operatorname{Re}(Z)-(-\operatorname{Im}(Z))$ diagram). Therefore, the sensor behavior can be interpreted as an $R C$ equivalent circuit. Fig. 1 shows the calculated sensor capacitance change $\Delta C=C_{0}-C_{\text {H2O }}$, which denotes the difference between the planar substrate capacitance and the capacitance of the droplet-covered device. At $t_{0}$, water drops are applied to the IDE and the capacitance increases. The moment, when condensate forms or water drops impinge on the sensor element is evident. The capacitance change depends on the diameter $d$ of the water covered IDE area, which increases from curve $\mathrm{A}$ to $\mathrm{E}$, respectively. At $t_{2}$, the capacitance reaches again the initial value, indicating that the sensor element is free of condensate and all condensate is evaporated.

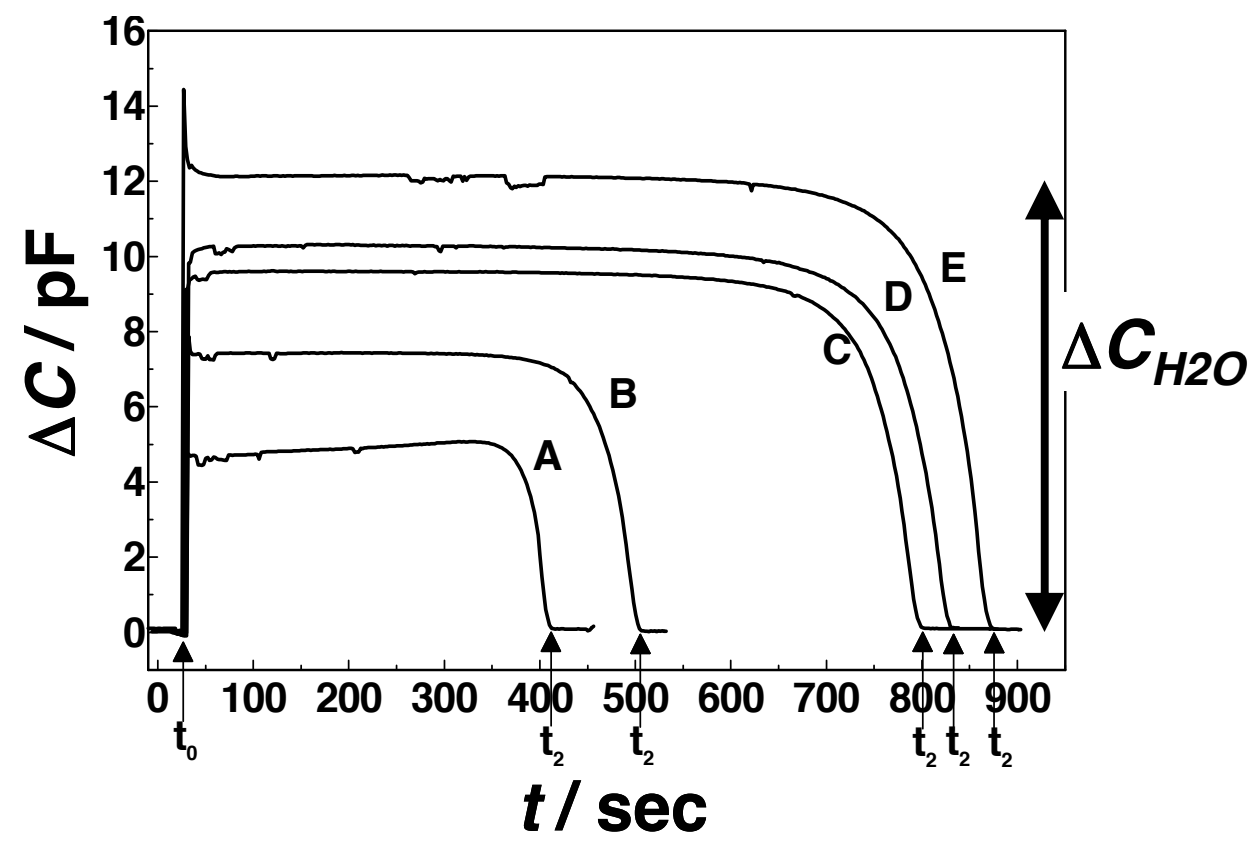

Fig. 1 Humidity sensor signal for different drop sizes

The $\Delta C$ vs. $d^{2}$ plot, illustrated in Fig. 2, shows a linear behaviour with a slope of $1.69 \mathrm{pF} / \mathrm{mm}^{2} . d$ is the diameter of the water droplet on the IDC surface, determined by microscope images. The relation $\Delta C \propto$ $d^{2}$ can be explained by a theoretical model which depends on the water covered area $\left(1 / 4 \pi d^{2}\right)$, the capacitance in air $C_{0}$, the IDE area $A_{\mathrm{IDE}}$, and the dielectric constants of air and water. 


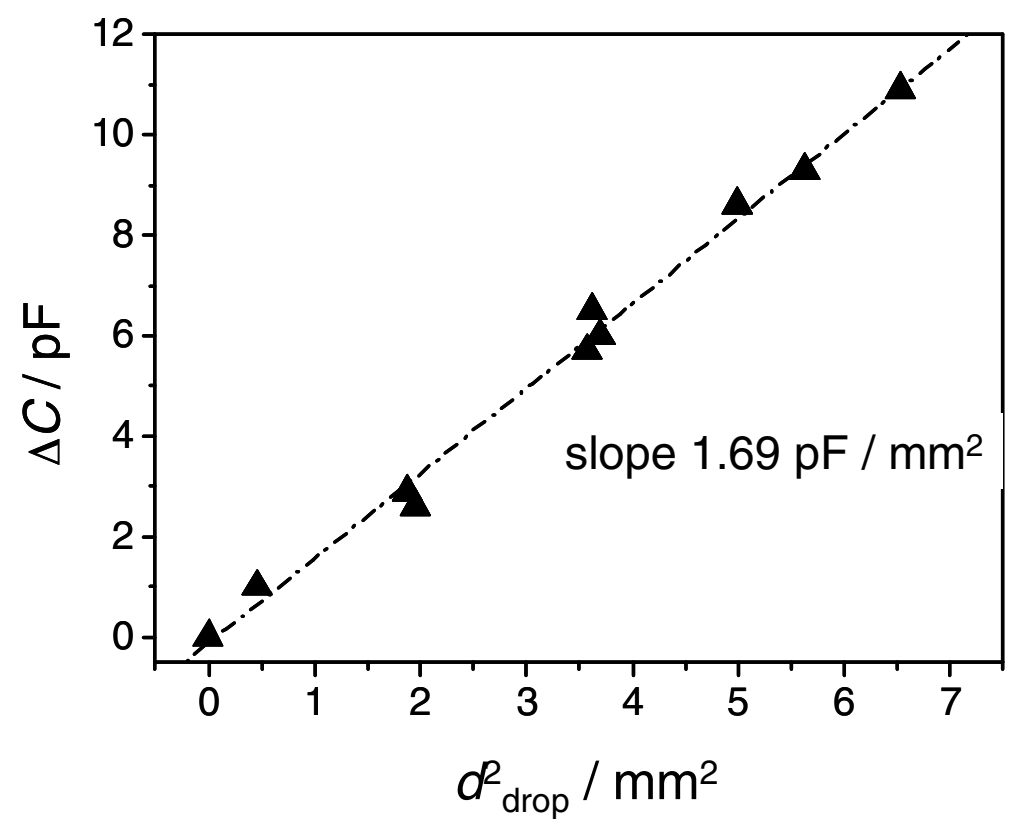

Fig. 2 Capacitance change $\Delta C$ as a function of the drop diameter $d$

The result of an engine test in real exhaust gas during engine cold start is shown in Fig. 3. $\Delta C$ increases immediately after engine start $\left(t_{0}\right)$ and reaches a maximum at $t_{1}$. During this period, condensate is formed on the sensor element yielding in a $\Delta C$ increase. A temperature sensor signal confirms this. The temperature reaches a plateau at $t_{1}$, indicating that the dew point is reached. After $t_{1}$, no more condensate is formed. The condensed water starts to evaporate and the sensor signal decreases until it reaches again the initial value at $t_{2}$, which means that the sensor element is free of condensate. The temperature curve confirms the above-said. As long as there is water on the sensor, the temperature shows a plateau. As soon as the sensor is water free, the temperature increases again.

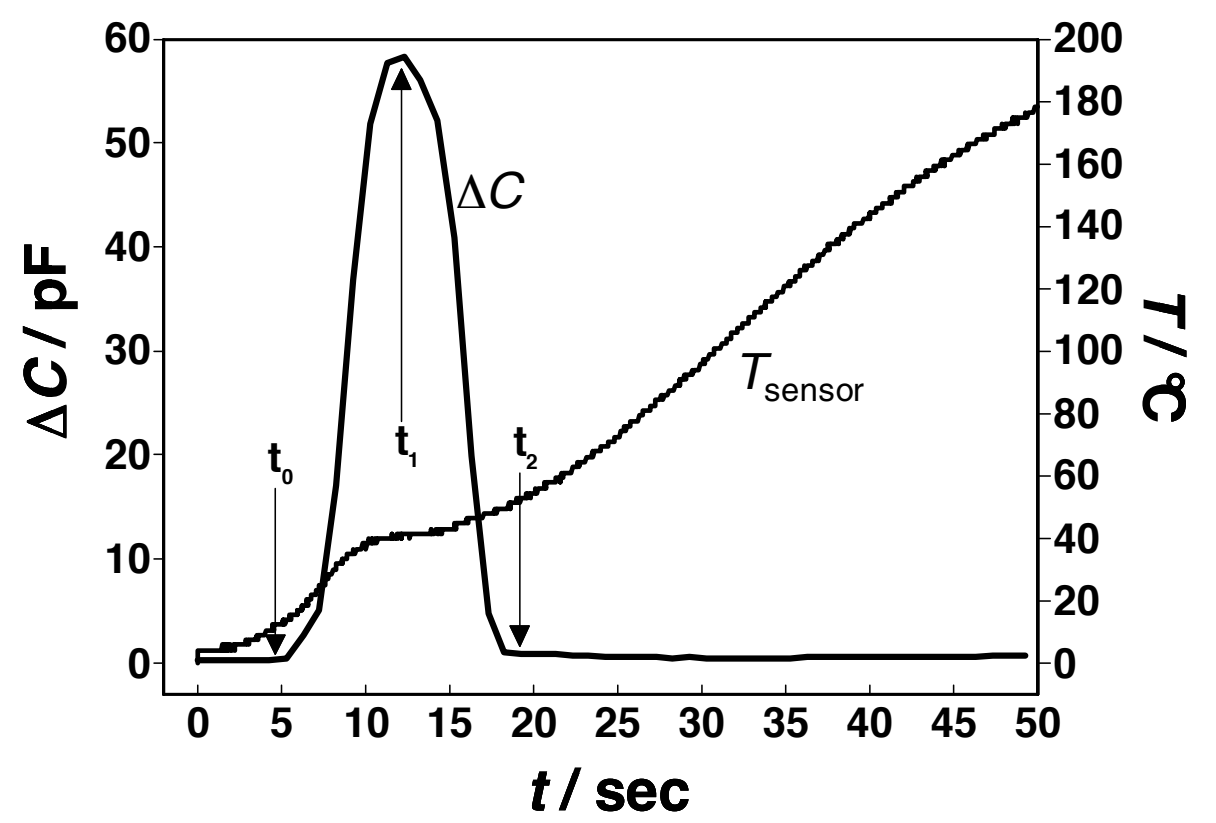

Fig. 3 Results of an engine cold start test 
Since $t_{1}$ and $t_{2}$ are correlated with the formation of condensate on the sensor element itself, the observed behavior can be used to improve the strategy of exhaust gas sensor heating. Compared to the $\Delta C$-signal, the temperature is less valuable for detecting the end of the dew point. By integrating an IDE structure on a planar $\lambda$-sensor and evaluating the course of the $\Delta C$-signal, an earlier sensor heat-up start can be realized, since no safety margins have to be considered.

\section{Conclusion}

The $\mathrm{H}_{2} \mathrm{O}$ sensing structure applied on a ceramic element provides the possibility to determine the water condensation process in the exhaust system during engine cold start. The sensor heating strategy can be improved to avoid sensor damage due to condensed water and water splash.

\section{References}

1. J. Riegel, H. Neumann, H.-M. Wiedenmann; Solid State lonics, 152-153 (2002), pp 783-800.

2. M.R. Busch, P. Hawig; German Patent Specification DE 102004020139 (2004). 